\title{
ADJACENT BEAMS RESOURCE SHARING TO SERVE HOT SPOTS: A RATE-SPLITTING APPROACH
}

\author{
Tomás Ramírez ${ }^{1}$, Carlos Mosquera ${ }^{2}$, Màrius Caus $^{3}$, Adriano Pastore $^{4}$, Nader Alagha $^{5}$, and Nele \\ Noels $^{6}$ \\ ${ }_{1,2}$ AtlanTTic research center, University of Vigo, Galicia, Spain \\ ${ }^{3,4}$ Centre Tecnològic de Telecomunicacions de Catalunya, Castelldefels, Barcelona, Spain \\ ${ }^{5}$ European Space Agency Technical Research Center (ESTEC), Noordwijk, The Netherlands \\ ${ }^{6}$ DIGCOM research group, TELIN Department, Ghent University, Ghent, Belgium
}

\begin{abstract}
This work addresses the uneven traffic demand scenario in multi-beam satellite systems, in which a hot-spot beam is surrounded by cold beams. After partitioning the hotspot beam in different sectors, resource pulling from cold neighbouring beams is allowed following an aggressive frequency-reuse scheme. As a consequence, the level of the co-channel interference within the hot-spot beam increases. A scheme known as Non-Coherent Rate-Splitting (NCRS) is employed to cope with this interference, based on the exclusive use of magnitude channel state information at the transmitter (CSIT). The receiver complexity is increased with respect to full CSIT precoding schemes, which are considered for benchmarking purposes. Different NCRS strategies are analyzed and compared with several partial and full CSIT schemes. The proposed solution not only shows an improvement with respect to partial CSIT benchmarks, but also displays a competitive performance against full CSIT precoders.
\end{abstract}

\section{Introduction}

The ever growing demand of data throughput and the limited available bandwidth for satellite communications has created the necessity of more spectrally efficient techniques, which involves the adoption of aggressive frequency reuse schemes. If more aggressive reuse schemes such as 1-color or 2-color are considered, the performance of single user detection (SUD) can suffer due to the increased level of co-channel interference in the satellite forward link from the gateways to the end user terminals. (see Fig. 1). In consequence, additional complexity needs to be implemented at the transmit gateway, receive terminals, or both. Precoding is one of the most promising techniques in the forward link to cope with co-channel interference in multibeam satellites. As main drawbacks, it requires a permanent reporting of the channel state information (CSI) to the gateway, and synchronism across the different beams. In [1], a multi-user detector (MUD) approach is considered to avoid these drawbacks and to only work with partial CSI at the transmitter (CSIT), e.g. the channel strength knowledge, at the cost of increasing the receiver complexity. Under similar partial CSIT constraints, in [2] and [3] a rate-splitting approach named
Non-Coherent Rate-Splitting (NCRS) was introduced to cope with the interference of neighboring beams. NCRS splits the messages into private and public components, and performs successive interference cancellation (SIC) at the receive terminals to extract messages that use the same time and frequency resources.

The uneven traffic scenario, with all the traffic demand concentrated in a single beam, has been recently studied in $[1],[4]$ and [5]. This scennario is usually referred to as hot spot (HS). Assuming partial CSIT, the MUD technique from [1] presents a significant improvement with respect to the 4-color Frequency Division Multiplexing (FDM) baseline, where terminals perform single-user detection (SUD) and no resource sharing among beams is performed. However, if resource pulling from neighbouring beams is allowed [4], adjacent beam resource sharing (ABRS) presents significant improvement in the capacity in comparison to conventional multi-beam systems without resource sharing. As presented in [1], the ABSR with 3 -color scheme even offers slight improvement over the MUD techniques without increasing the complexity of the receivers (using SUD) and without significant increase in the gateway uplink frequency (compared to 1 or 2-color schemes). On the other side, precoding techniques are analyzed in a Hot Spot (HS) scenario with full CSIT [5]. In addition to full CSIT, the performance of precoding is boosted by assuming a flexible payload and double polarization per beam. Diverse flavours of precoding are presented in [5] by following different optimization criteria. The so-called performance precoding presents the highest sum-rate after numerical optimization of the precoder coefficients, at the cost of introducing high imbalance in the rates assignment. A trade-off between fairness and sumrate is also pursued in this reference by changing the optimization metric.

The current paper extends the work in [4], by applying rate splitting ideas to a HS scenario with the adoption of aggressive frequency reuse patterns. NCRS has already proved a significant improvement with respect to the 4-color FDM benchmark, achieving up to $20 \%$ for low signal-to-noise ratio (SNR) cases in a uniform traffic scenario [3]. Nevertheless, it is yet to be confirmed whether NCRS can present an improvement over the previous techniques for HS. In order to quantify the potential 
improvement, both partial and full CSIT benchmarks are considered. Caution is also required when comparing different schemes due to the varying degrees of complexity at the receivers. Some comments will be made on the required complexity at the receive terminals, which will be assumed to operate ideally without any implementation losses.

The rest of the paper is organized as follows. In Section II, the satellite system model is introduced. Next, NCRS is presented in Section III. After that, the simulation results are shown in Section IV and some conclusions are given in Section $\mathrm{V}$.

Notation: Upper (lower) boldface letters denote matrices (vectors). (. $)^{H}, \mathbf{I}_{N}$ denote Hermitian transpose and $N \times N$ identity matrix, respectively. $\mathbb{E}[\cdot]$ is the expected value operator.

\section{System Model}

In this work we focus on the forward link of a multibeam satellite communication system. More specifically, we focus on a Hot-Spot scenario in which a central beam is surrounded by six other beams which have none or negligible traffic. To keep the analysis simple, the second ring and outer rings beams are not considered. Assuming perfect synchronization, an ideal feeder link, a single feed per beam payload architecture, and neglecting non-linear effects in the satellite payload, the values received by $M$ interfering users at a given time instant can be written as

$$
\mathbf{y}=\mathbf{H x}+\mathbf{w}
$$

where $\mathbf{y} \in \mathbb{C}^{M \times 1}$ is the vector of received signals given by $\mathbf{y}=\left[\begin{array}{llll}y_{1}[k] & y_{2}[k] & \ldots & y_{M}[k]\end{array}\right]^{T}, \mathbf{H} \in \mathbb{C}^{M \times M}$ is the channel matrix, $\mathbf{x} \in \mathbb{C}^{M \times 1}$ is the vector of transmitted signals given by $\mathbf{x}=\left[\begin{array}{llll}x_{1}[k] & x_{2}[k] & \ldots & x_{M}[k]\end{array}\right]^{T}$ and $\mathbf{w} \in \mathbb{C}^{M \times 1}$ is the noise vector given by $\mathbf{w}=\left[\begin{array}{llll}w_{1}[k] & w_{2}[k] & \ldots & w_{M}[k]\end{array}\right]^{T}$. To keep the notation simple, the time index is dropped from now on. All the antenna feeds transmit with the same power, so that $\mathbb{E}\left[\mathbf{x x}^{\mathrm{H}}\right]=\frac{P}{M} \mathbf{I}_{M}$ for a total power $P$. The samples of the Additive White Gaussian Noise (AWGN) vector $\mathbf{w}$ are statistically independent, i.e. $\mathbb{E}\left[\mathbf{w} \mathbf{w}^{\mathrm{H}}\right]=$ $\frac{\sigma^{2}}{\mathrm{C}} \mathbf{I}_{M}$, where $\mathrm{C}$ is the number of frequency colors in the system.

The $(q, m)$ th entry of the matrix $\mathbf{H}$ is given by:

$$
h_{q m}=e^{j \phi_{q m}} \frac{\sqrt{G_{R} G_{q m}}}{4 \pi d_{q} / \lambda} .
$$

Here, the term $G_{R}$ refers to the receive antenna gain and $G_{q m}$ represents the transmit antenna gain from beam $m$ to the $q$-th user terminal. As for the rest of the terms, $\lambda$ is the carrier wavelength, $\phi_{q m}$ is the phase rotation introduced by the channel and $d_{q}$ is the distance from the satellite to the $q$-th user.

Since the rate-splitting approach only uses magnitude information, we introduce also the link strength between the $q$ th feed and the $m$ th receiver:

$$
\gamma_{C, q m}=\frac{\frac{P}{M}\left|h_{q m}\right|^{2}}{\sigma^{2} / \mathrm{C}} .
$$

\subsection{Hot-Spot scenario}

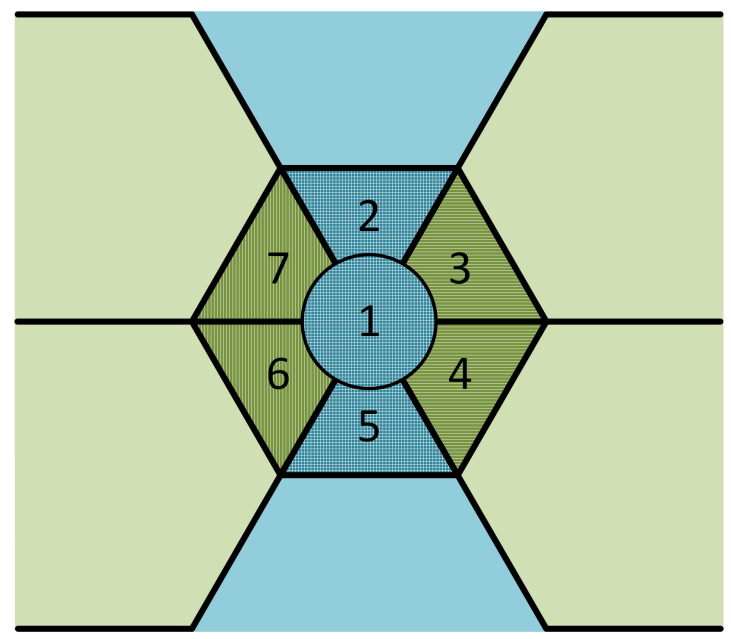

(a) 2-color scheme $\mathrm{A}$

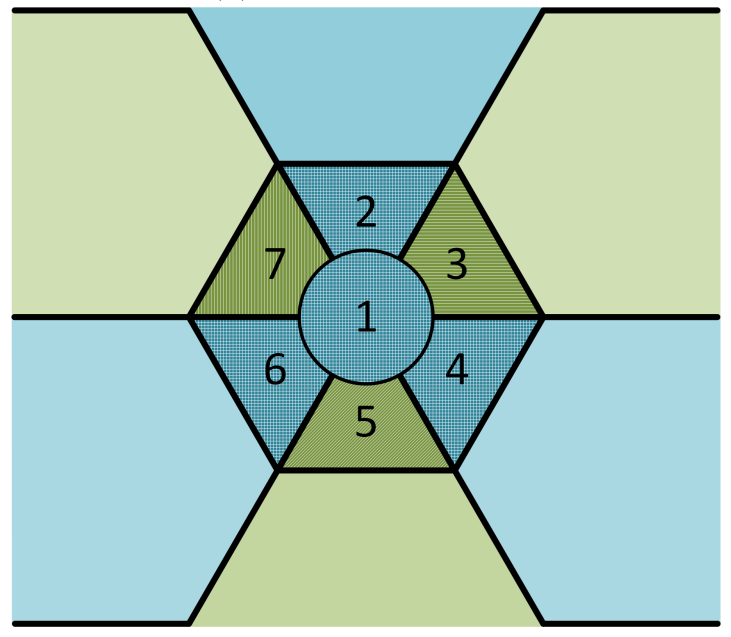

(b) 2-color scheme B

Fig. 1 Mapping of colors to the central beam for 2-color frequency reuse schemes.

The resources of the adjacent beams are shared with the hot spot beam in order to satisfy the traffic demand as in [4]. To this end, the hot spot beam is divided into seven sectors which are served by the respective beams. Figs. 1 and 2 sketch the boundaries of the sectors for different frequency schemes, with different colors denoting different frequency bands. Examples of 3 -color and 4-color frequency allocations are given in Fig. 2 according to [1]. The radius of the inner zone is determined by a feed gain threshold, $G_{t h}$, relative to the maximum feed gain of the beam* ${ }^{*}$.

${ }^{*}$ The relative gain $G_{t h}$ is used for theoretical purposes. In an actual satellite system, it would be more practical to use instead 


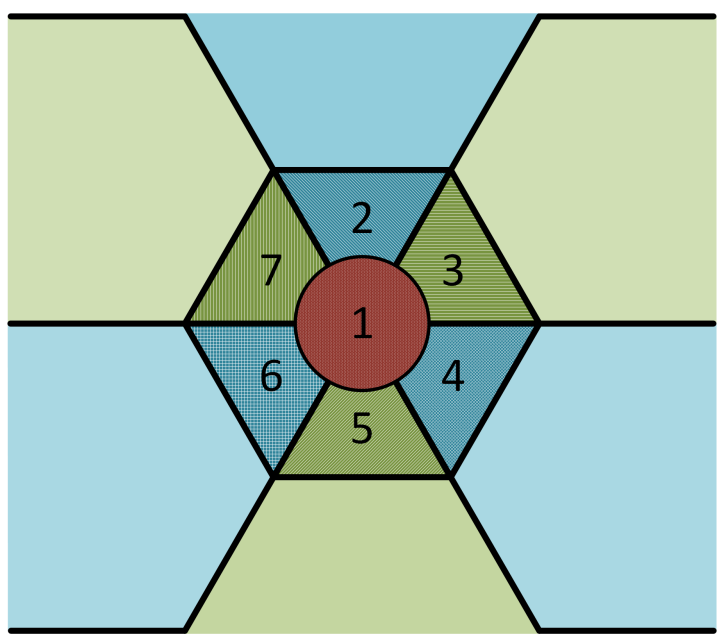

(a) 3-color scheme

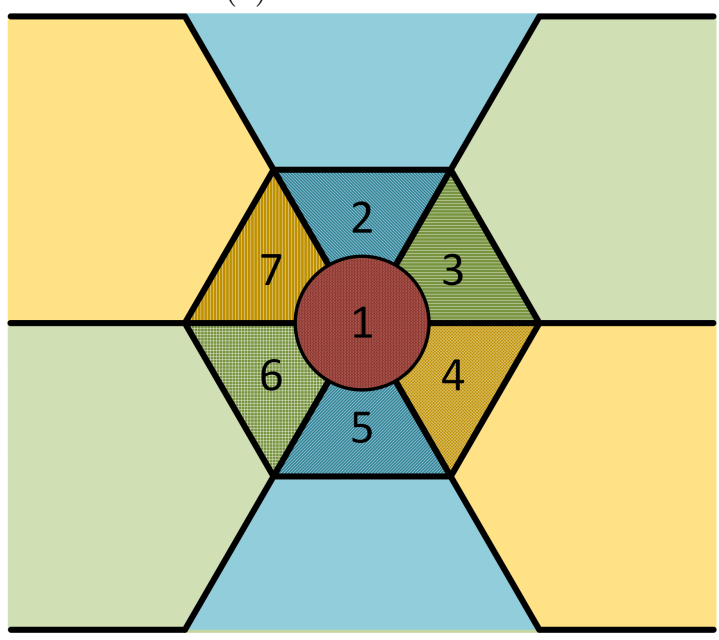

(b) 4-color scheme

Fig. 2 Mapping of colors to the central beam for different frequency reuse schemes.

The more aggressive 2-color schemes from Fig. 1 gives rise to a much higher amount of interference; the lack of full CSIT at the gateway, with the only knowledge of the magnitude of the received signals from the different beams, makes us consider a non-orthogonal rate splitting scheme known as NCRS and presented in [2]. NCRS can operate with groups of two or more simultaneous users using the same frequency resources, and does not require tight synchronization requirements among the superimposed signals. A SIC strategy is employed at the receiver side to get the most out of the downlink flow. The beam grouping depicted in Fig. 1(a) is such that two pairs of beams (in green color) and a group of three beams (in blue color) are handled separately. In Fig. 1(b) NCRS is used to exploit the group of 4 beams (in blue color), with the users in the other three green zones performing SUD. As discussed in the next section, partial CSIT benchmarks will be also simulated for both 3 -color and 4-color reuse schemes from

signal to interference and noise (SINR) measurements at the receiver side which are fedback to the gateway.
Fig. 2 based on the ABRS approach of [4]. We will also consider full CSIT benchmarking for the full frequency reuse (FFR) scenario, in the form of precoding.

\subsection{Satellite payload}

The payload constraints are the same as in [1] and [4], where conventional payload structures for multi-beam satellites are assumed. For the sake of simplicity, perfect synchronization across beams is assumed in this work, although it is not required in the case of ABRS and NCRS [6]. However, in the latter case, the lack of synchronism demands more complexity from the receiver if applying joint decoding of the received signals.

\section{Non-Coherent Rate-Splitting (NCRS)}

Inspired by the Generalized Degrees of Freedom(GDoF) framework in [7], NCRS is presented in [2] and [3] to cope with the co-channel interference by using rate-splitting. The transmitters send two kind of messages: private and public. The former is decoded by and intended for one of the users and the latter is decoded by all the users. The amount of power allocated to each message is controlled by a variable $\lambda_{i}$, which needs to be optimized for a given quality of service (QoS) criterion. For a two user scenario the transmitted signals are expressed as

$$
\begin{aligned}
& x_{1}=\sqrt{\frac{P}{2}\left(1-\lambda_{1}\right)} x_{c_{1}}+\sqrt{\frac{P}{2} \lambda_{1}} x_{p_{1}} \\
& x_{2}=\sqrt{\frac{P}{2}\left(1-\lambda_{2}\right)} x_{c_{2}}+\sqrt{\frac{P}{2} \lambda_{2}} x_{p_{2}}
\end{aligned}
$$

with $\mathbb{E}\left[\begin{array}{ll}\left|x_{1}\right|^{2} & x_{1} x_{2}^{*} \\ x_{2} x_{1}^{*} & \left|x_{2}\right|^{2}\end{array}\right]=\frac{P}{2} \mathbf{I}$.

The public message is transmitted by sending two public messages $x_{c_{1}}$ an $x_{c_{2}}$ which can be jointly or successively decoded. At the receiver side, the public messages are decoded first by treating the private messages $x_{p_{1}}$ and $x_{p_{2}}$ as noise. After subtracting the public message, the private messages are decoded. In this paper, joint decoding (JD) of the public messages is assumed and thereby, the NCRS receiver involves JD and successive interference cancellation (SIC). The application of NCRS to more than two users increases substantially the complexity of the process to optimize the weights of the different public messages, and requires more cancellation stages at the receivers. As a suboptimal extension, we just consider a public message component $x_{c_{i}}$ for each user. For the 3 -user case, NCRS signals would read as

$$
\begin{aligned}
& x_{1}=\sqrt{\left(1-\lambda_{1}\right) P} x_{c_{1}}+\sqrt{\lambda_{1} P} x_{p_{1}} \\
& x_{2}=\sqrt{\left(1-\lambda_{2}\right) P} x_{c_{2}}+\sqrt{\lambda_{2} P} x_{p_{2}} \\
& x_{3}=\sqrt{\left(1-\lambda_{3}\right) P} x_{c_{3}}+\sqrt{\lambda_{3} P} x_{p_{3}}
\end{aligned}
$$


whereas the corresponding 4-user NCRS signals are given by

$$
\begin{aligned}
& x_{1}=\sqrt{\left(1-\lambda_{1}\right) P} x_{c_{1}}+\sqrt{\lambda_{1} P} x_{p_{1}} \\
& x_{2}=\sqrt{\left(1-\lambda_{2}\right) P} x_{c_{2}}+\sqrt{\lambda_{2} P} x_{p_{2}} \\
& x_{3}=\sqrt{\left(1-\lambda_{3}\right) P} x_{c_{3}}+\sqrt{\lambda_{3} P} x_{p_{3}} \\
& x_{4}=\sqrt{\left(1-\lambda_{4}\right) P} x_{c_{4}}+\sqrt{\lambda_{4} P} x_{p_{4}} .
\end{aligned}
$$

Note that the signals indexes in (7) and (8) are expressed in a general way, and do not hold any relation to the sector indexes in Figs. 1 and 2.

Therefore, for an $N$-user NCRS scenario, $N+1$ rates have to be selected, splitted as $N$ private rates and one public rate. If $W$ denotes the available bandwidth, the $i$ th user private rate is given by

$$
R_{p_{i}}=\frac{W}{2} \log _{2}\left(1+\frac{\lambda_{i} \gamma_{2, i i}}{1+\sum_{\substack{m=1 \\ m \neq i}}^{N} \lambda_{m} \gamma_{2, i m}}\right)
$$

whereas the rate of the public message at the $i$ th receiver reads as

$$
R_{c_{i}}=\frac{W}{2} \log _{2}\left(1+\frac{\sum_{m=1}^{N}\left(1-\lambda_{m}\right) \gamma_{2, i m}}{1+\sum_{m=1}^{N} \lambda_{m} \gamma_{2, i m}}\right)
$$

The public message is to be decoded by all users, so that its rate is given by

$$
R_{c}=\min _{j} R_{c_{j}}
$$

Since the public message is decoded by all the users, it can be made of the aggregation of messages addressed to the different users. There is not any restriction in how to share the public information among users. The user rates are given

$$
R_{m}=R_{p_{m}}+\beta_{m} R_{c}
$$

where $\beta_{m}$ is the portion of the public message which is assigned to the $m$ th user and $\sum_{m=1}^{N} \beta_{m}=1$. In this paper, we are going to assume that the criterion of the $\beta_{m}$ weights is to assign the portions in such a way that user rates are as evenly distributed as possible.

\subsection{Rate optimization}

$N$ optimization problems need to be solved, one for each user setting the minimum rate of the public message in
(10). From this relation, we have that $R_{c}=R_{c_{i}}$ if, for $j=$ $\{1,2 \ldots, N\}$ and $j \neq i$,

$$
L_{0 i j}+\sum_{m=1}^{N} L_{m i j} \lambda_{m} \geq 0
$$

with

$$
\begin{aligned}
\alpha_{i} & =1+\sum_{s=1}^{N} \gamma_{2, i s} \\
L_{0 i j} & =\frac{1}{\alpha_{i}}-\frac{1}{\alpha_{j}} \\
L_{m i j} & =\frac{\gamma_{2, i m}}{\alpha_{i}}-\frac{\gamma_{2, j m}}{\alpha_{j}} .
\end{aligned}
$$

With this, the $N$ non-convex sub-problems $P_{i}$ which need to be solved to maximize the sum-rate are expressed as

$$
\begin{aligned}
& \left(P_{i}\right) \underset{0 \leq \lambda_{1}, \ldots, \lambda_{N} \leq 1}{\operatorname{argmax}} \quad R_{c_{i}}+\sum_{m=1}^{N} R_{p_{m}} \\
& \text { s.t. } \quad L_{0 i j}+\sum_{m=1}^{N} L_{m i j} \lambda_{m} \geq 0 \\
& j=1,2, \ldots, N, j \neq i \text {. }
\end{aligned}
$$

This sum-rate optimization cannot guarantee a fair assignation of rates. In order to prevent some users from being drastically underserved, the harmonic mean can be used as optimization metric:

$$
\begin{array}{cc}
\left(P_{i}\right) \underset{0 \leq \lambda_{1}, \ldots, \lambda_{N} \leq 1}{\operatorname{argmax}} & \frac{N}{\sum_{m=1}^{N} \frac{1}{R_{m}}} \\
\text { s.t. } & L_{0 i j}+\sum_{m=1}^{N} L_{m i j} \lambda_{m} \geq 0 \\
& j=1,2, \ldots, N, j \neq i .
\end{array}
$$

For both optimization problems, the different maximization sub-problems can be pursued by applying a sequential quadratic programming method [8]. The optimal solution will be the best among the obtained solutions.

\section{Competitive techniques}

In order to measure the potential improvement of NCRS in a HS scenario, other techniques, either using full or partial CSIT, will be also implemented for benchmarking purposes.

\subsection{Partial CSIT}

In [4], ABRS has shown a potential improvement with respect to the MUD technique in [1], requiring also a simpler receiver. Therefore, ABRS is chosen as a partial CSIT benchmark and will be simulated for the 3-color and 4color schemes. The main idea of ABRS is to pull the adjacent cold beam resources to cope with the traffic demand in the HS beam. Thus, the system capacity can be increased without requiring any additional complexity at the 
satellite or the receiver with respect to traditional FDM. Both ABRS and NCRS make use of the magnitude CSIT with some differences on how this information is reported; as detailed earlier, NCRS operates with the knowledge of the disaggregated power values (3) in the form of SNR and interference to noise ratios (INR), whereas ABRS only needs the signal to interference and noise ratio (SINR) at the receiver. The corresponding ABRS rates for the $i$ th user in the 3-color and 4-color cases are given by

$$
R_{i}=\frac{W}{3} \log _{2}\left(1+\frac{\gamma_{3, i i}}{1+\sum_{m \in \mathcal{S}_{i}}^{N} \gamma_{3, i m}}\right)
$$

and

$$
R_{i}=\frac{W}{4} \log _{2}\left(1+\frac{\gamma_{4, i i}}{1+\sum_{m \in \mathcal{G}_{i}}^{N} \gamma_{4, i m}}\right),
$$

respectively, where $\mathcal{S}_{i}$ and $\mathcal{G}_{i}$ are the subset of beam indexes which are treated as noise by the $i$ th receiver at each case.

\subsection{Full CSIT}

In [5], different precoders are analyzed in a full frequency reuse HS scenario. For comparison purposes, the performance precoding in [5] will be simulated. The inputoutput relation is expressed as

$$
\mathbf{y}=\mathbf{H F x}+\mathbf{w}
$$

with $\mathbf{F x}$ the precoded symbols. The precoding matrix $\mathbf{F}$ is obtained as the solution of the following non-convex optimization problem:

$$
\begin{array}{ll}
\mathbf{F}_{\text {opt }}=\underset{F}{\operatorname{argmax}} & \sum_{m=1}^{N} R_{m} \\
\text { s.t. } & \left\|\boldsymbol{F}_{i}\right\|^{2} \leq \frac{P}{M} \\
& R_{m}=W \log _{2}\left(1+\frac{\left|H_{m} f_{m}\right|^{2}}{\sigma^{2}+\sum_{l \neq m}\left|H_{l} f_{l}\right|^{2}}\right)
\end{array}
$$

with $\mathbf{F}_{i}$ and $\boldsymbol{f}_{i}$ the $i$ th row and the $i$ th column of the matrix $\mathbf{F}$, respectively, and $\mathbf{H}_{i}$ the $i$ th row of the channel matrix H.

In addition, the closed form MMSE precoder will be also simulated. It reads as

$$
\mathbf{F}=\sqrt{v} \mathbf{H}^{\mathrm{H}}\left(\mathbf{H} \mathbf{H}^{\mathrm{H}}+\frac{M}{P} \sigma^{2} \mathbf{I}\right)^{-1}
$$

where $v$ is a scalar such that the power constraint per feed is satisfied, and it is expressed as

$$
v=\frac{P / M}{\max \left(\operatorname{diag}\left(\mathbf{H}^{\mathrm{H}}\left(\mathbf{H} \mathbf{H}^{\mathrm{H}}\right)^{-2} \mathbf{H}\right)\right)} .
$$

\section{Numerical Results}

The performance of the considered techniques has been tested for the different color schemes in Figs. 1 and 2, and the system parameters included in Table 1 . The simulations are parameterized by $G_{t h}{ }^{\dagger}$, the radius of the inner zone. For each different value of $G_{t h}, 5,000$ Monte Carlo realizations have been run, with uniform distribution of users in each HS section. The total average spectral efficiency is displayed in Fig. 3 for all techniques. Please note that perfect CSI is being assumed for the precoding techniques, and perfect cancellation at the receive terminals when applying SIC in the case of NCRS.

Table 1 System Parameters.

\begin{tabular}{cc}
\hline \multicolumn{2}{c}{ Satellite foward link } \\
\hline Diagram pattern & Provided by ESA [1] \\
\hline Number of beams & 7 \\
\hline Number of feeds & 20 \\
\hline Feed synchronization & Perfect synchronization \\
\hline Frequency band [GHz] & $67 \mathrm{dBW}$ \\
\hline EIRP/beam & 19 \\
\hline Far Field Noise Power Ratio (NPR) & 0.65 \\
\hline Receiver Parameters & $0.6 \mathrm{~m}$ \\
\hline Receiver antenna efficiency & $280^{\circ} \mathrm{K}$ \\
\hline Receiver antenna diameter & $310^{\circ} \mathrm{K}$ \\
\hline Receiver cloud noise temperature & $45^{\circ} \mathrm{K}$ \\
\hline Receiver terminal noise temperature & $2 \mathrm{~dB}$ \\
\hline Receiver ground noise temperature & Ideal cancellation \\
\hline LNB Noise Figure & \\
\hline Interference cancellation &
\end{tabular}

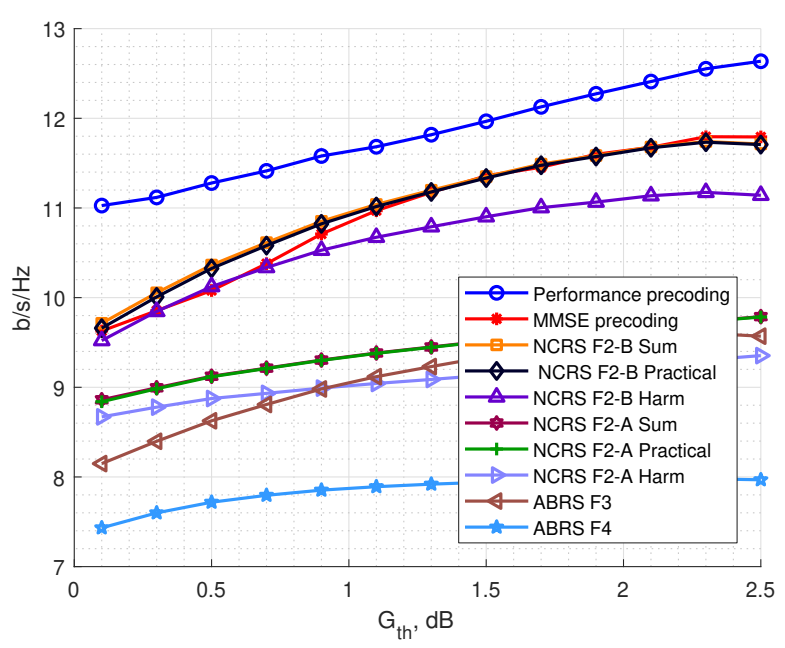

Fig. 3 Aggregated spectral efficiency versus gain threshold.

As expected, the maximum rate is achieved after the optimization of the precoding coefficients in (21), by the

${ }^{\dagger}$ Note that for $G_{t h}=0$ the inner zone would collapse into a point. 
so-called performance precoder. MMSE performance compares with that of NCRS-B, whereas ABRS under three colors is similar to NCRS-A. Interestingly, the optimization of $\left\{\lambda_{i}\right\}, i=1,2,3,4$ in (16) -to maximize the overall throughput of the group of 3 and 4 beams in Fig. 1- is such that most of the content of the message sent from the central beam should be public, whereas most of the content of the messages sent from the three green peripheral beams should be private. Therefore, some complexity can be saved by making a practical design with $\left(\lambda_{1}, \lambda_{2}, \lambda_{3}\right)=(0,1,1)$ and $\left(\lambda_{1}, \lambda_{2}, \lambda_{3}, \lambda_{4}\right)=(0,1,1,1)$ for the 3 -user and 4-user cases, respectively. This operation point, labeled as "Practical" in Fig. 3, suffers only from a marginal degradation, within the range of $0-2 \%$. The corresponding signals for the group of 3 beams (2-color scheme B) are written now as

$$
\begin{aligned}
& x_{1}=\sqrt{P} x_{c_{1}} \\
& x_{2}=\sqrt{P} x_{p_{2}} \\
& x_{3}=\sqrt{P} x_{p_{3}}
\end{aligned}
$$

whereas for the group of 4 beams (2-color Scheme A) are written as

$$
\begin{aligned}
& x_{1}=\sqrt{P} x_{c_{1}} \\
& x_{2}=\sqrt{P} x_{p_{2}} \\
& x_{3}=\sqrt{P} x_{p_{3}} \\
& x_{4}=\sqrt{P} x_{p_{4}}
\end{aligned}
$$

where $x_{1}$ is the transmitted signal by the central beam. Note that this practical design avoids the optimization step, and reduces the complexity of the receiver: JD is not needed, and only a one SIC stage is applied. On the other side, some flexibility in the allocation of the rates is lost as the $\lambda_{i}$ values are fixed.

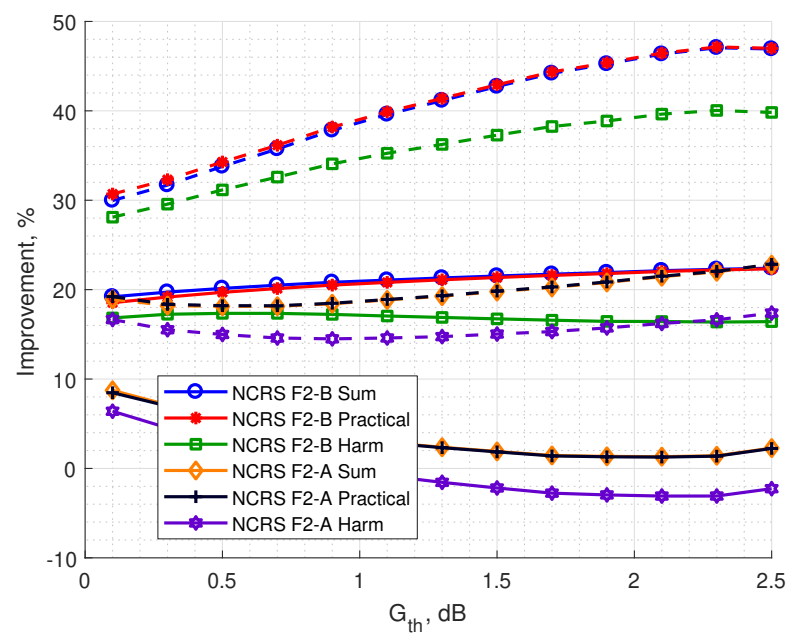

Fig. 4 Relative sum-rate performance between noncoherent schemes (NCRS) and partial CSIT benchmarks. Solid curves report the improvement over 3-color ABRS. Dashed lines report the improvement over 4-color ABRS.

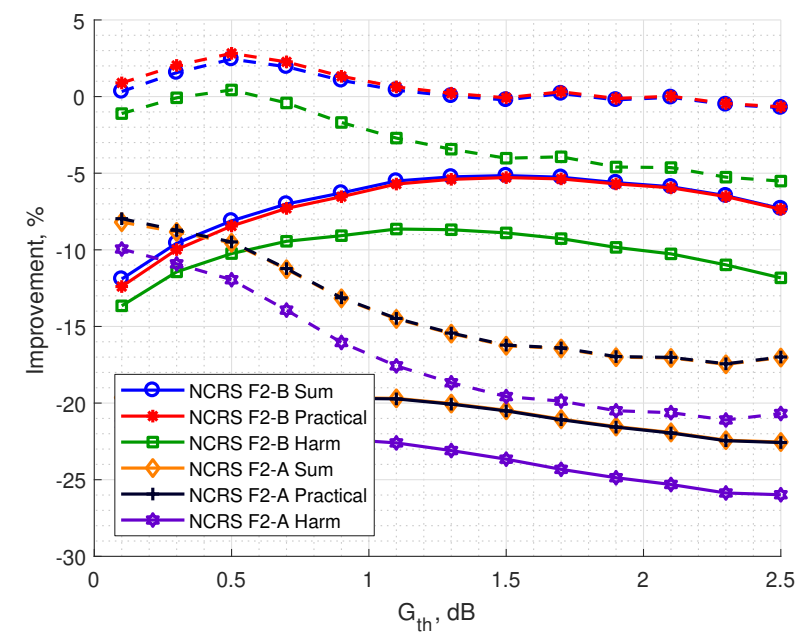

Fig. 5 Relative sum-rate performance between noncoherent schemes (NCRS) and full CSIT precoding. Dashed curves report the improvement over MMSE precoding. Solid lines report the improvement over performance precoding.

A close look at the comparison between partial CSIT schemes is depicted in Fig. 4. The improvement is significant in the case of the 2-color scheme B. However, the more modest improvement of the 2-color scheme A can be more significant from a satellite payload complexity point of view. In [3], the 2-color scheme A was simulated in a uniform traffic distribution scenario, with an improvement over the traditional 4-FDM benchmark. Without requiring any change in the frequency of the beams, NCRS is able to provide some improvement for both the uniform and uneven traffic scenario. This might not be the case for the 2-color scheme B, with more adjacent beams with the same color and, in consequence, higher co-channel interference.

NCRS is also competitive when compared with precoding at the gateway, despite the use of a lower amount of CSI. Fig. 5 shows how the performance precoder [5] offers the best aggregate sum-rate with some margin over the others techniques, whereas NCRS and the MMSE precoder perform quite similarly. The design of the MMSE precoder is simple given the availability of a closed form expression (22). The performance precoding presents high complexity since an $N \times N$ matrix needs to be found after a non-convex optimization problem. In the case of NCRS the maximum number of elements to be optimized is $N$. On the other side, NCRS demands more complexity from the receive terminals as compared with the benchmark receivers.

In addition to the overall rate, how the bit rate is allocated to the different users is especially relevant. We have used the Jain's fairness index [9] to compare the rate allocation results of the different schemes. The results are shown in Fig. 6 for different values of $G_{t h}$. The use of full CSIT and a more complex optimization process, by employing the performance precoding technique in [5], can 


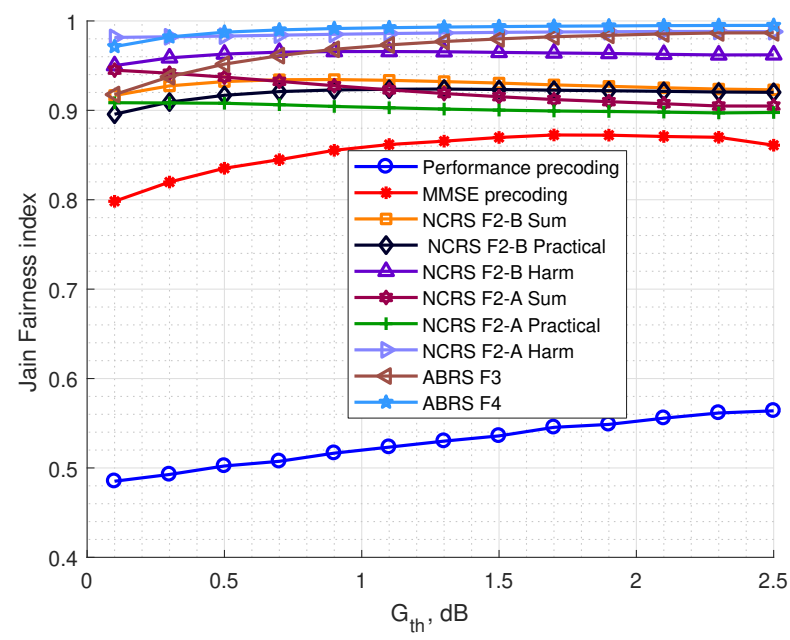

Fig. 6 Jain index versus gain threshold.

achieve a larger sum rate, at the cost of creating strong differences in the allocated rates. The required complex numerical optimization to obtain the optimal precoder in [5] can be alleviated by using the MMSE precoder, which is also more balanced when assigning rates, although still outperformed by ABRS and NCRS in terms of fairness.

The use of the harmonic mean criterion (17) serves to improve the fairness in NCRS, as concluded from Fig. 6, for a moderate degradation of the overall sum-rate (Fig. 3). This is further illustrated in Fig. 5, which displays the probability density function (pdf) of the rates for different NCRS optimization criteria, where $R_{i}$ represents the rate of users located at the $i$ th sector from Fig 1. Fig. 5 clearly depicts how the harmonic mean criterion guarantees a more fair allocation of user rates. This is especially significant for the user served at the central area of the HS, labeled as R1. Thus, by changing the optimization metric in NCRS, different sets of weights $\lambda_{i}$ are obtained.

Overall, the rate-splitting approach of NCRS outperforms the partial CSIT benchmarks and, surprisingly, can match and even surpass the MMSE precoder in some cases. On the other side, performance precoding presents the highest aggregated sum-rate as a result of the numerical maximization of the overall rate with full CSIT. The comparison between the different techniques is summarized in Table 2.

\section{Conclusions}

In this paper, a rate-splitting approach has been successfully tested in a Hot-Spot scenario. For this purpose, the work in [2] and [3] has been extended to embrace the HotSpot case analyzed in [4]. Since NCRS has been initially designed for a two user case, a simplified scaled version for three and four users has been introduced. NCRS is able to provide a modest improvement over the partial CSIT benchmarks [4], around $20 \%$ and $40 \%$ with respect to the 3 -color and 4-color ABRS, respectively, at the cost of increasing the receiver complexity. Somewhat surprisingly,

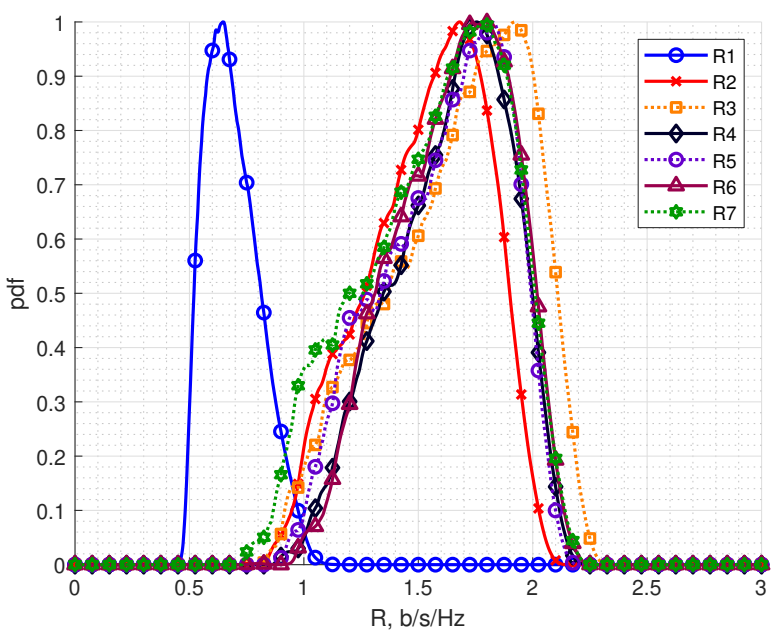

(a) 2-color B. NCRS with sum-rate optimization.

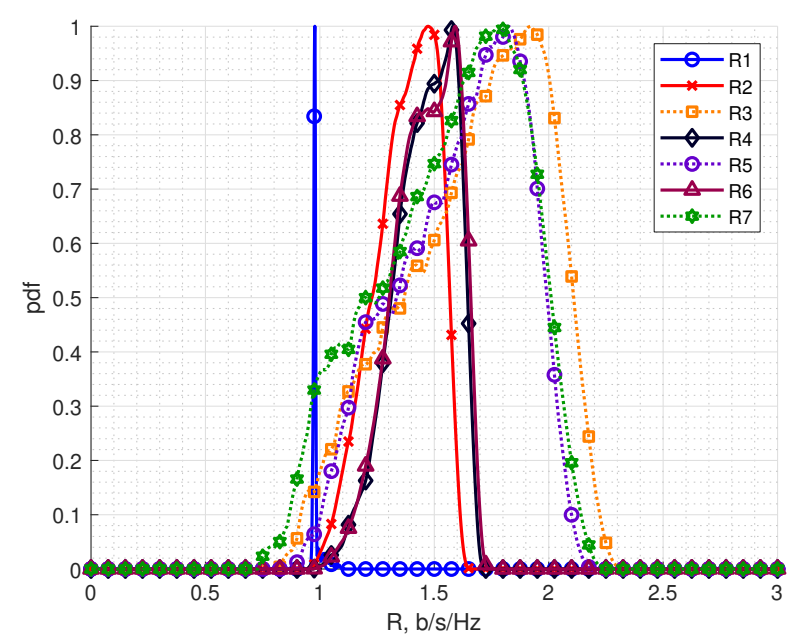

(b) 2-color B. NCRS with harmonic mean optimization.

Fig. 7 Different rate probability distribution functions for 2-color NCRS B. $G_{t h}=0.5$.

NCRS achieves competitive rates with respect to the full CSIT precoding benchmarks. The practical MMSE precoder shows a close performance with respect to NCRS, despite the different CSIT requirements. However, if the precoder is designed to maximize the sum rate [5], NCRS cannot match the performance. Nonetheless, this precoder assigns quite unevenly the rates among the users. Alternatively, when it comes to the fairness, NCRS seems to be more amenable to a fair allocation of rates than precoding, specially when the harmonic mean is the optimization criterion. It should be remarked that the capacity analysis in this work does not involve any modulation and coding nor practical implementation assumptions, which will undoubtedly impact the results in the system analysis. In this regard, 3-color ABRS seems to pose a good trade-off between performance and implementation simplicity.

\footnotetext{
$\ddagger$ Even though strict synchronism is not required, the time misalignment among beams will require more complexity at the receive
} 
Table 2 Main features of the compared techniques

\begin{tabular}{cccccccc}
\hline $\begin{array}{c}\text { Color } \\
\text { scheme }\end{array}$ & Technique & Sum-Rate & Fairness & $\begin{array}{c}\text { CSIT } \\
\text { requirement }\end{array}$ & $\begin{array}{c}\text { Receiver } \\
\text { complexity }\end{array}$ & $\begin{array}{c}\text { Optimization } \\
\text { complexity }\end{array}$ & $\begin{array}{c}\text { Beam } \\
\text { synchronization }\end{array}$ \\
\hline 4-colors & ABRS & Poor & Good & Partial (SINR) & Low & None & Not required \\
\hline 3 -colors & ABRS & Fair & Good & Partial (SINR) & Low & None & Not required \\
\hline 2 -colors & NCRS-A & Fair & Good & Partial (SNR,INR) & High & Fair & Not required ${ }^{\ddagger}$ \\
\hline 2 -colors & NCRS-B & Good & Good & Partial (SNR,INR) & High & Fair & Not required \\
\hline FFR & MMSE precoding & Good & Fair & Full & Low & None & Required \\
\hline FFR & Performance precoding & Very good & Poor & Full & Low & High & Required \\
\hline
\end{tabular}

\section{Acknowledgements}

This work has been supported by European Space Agency funded activity SatNEx IV CoO2-Part 1 WI 3 "Non-Orthogonal Superposition Techniques for MultiBeam Satellite Networks". The views of the authors of this paper do not reflect the views of ESA. This work was partially funded by the Agencia Estatal de Investigacion (Spain) and the European Regional Development Fund (ERDF) through the projects TERESA (TEC201790093-C3-1-R) and MYRADA (TEC2016-75103-C2-2-R). Funded also by the Catalan Government under project 2017-SGR-1479, and Galician Government under project ED431C 2017/53.

\section{References}

[1] R.D. Gaudenzi, N. Alagha, M. Angelone, and G. Gallinaro. 'Exploiting Code division Multiplexing with Decentralized Multiuser Detection in the Satellite Multibeam Forward Link'. International Journal of Satellite Communications and Networking, 36(3):239-276.

[2] M. Caus, A. Pastore, T. Ramírez, C. Mosquera, and N. Noels. 'Exploratory Analysis of Superposition Coding and Rate-Splitting for Multibeam Satellite Systems'. In 15th International Symposium on Wireless Communications Systems, Aug 2018.

[3] T. Ramírez, C. Mosquera, M. Caus, A. Pastore, M. Navarro, and N. Noels. 'Message-Splitting for Interference Cancellation in Multibeam Satellite Systems'. In 9th Advanced Satellite Multimedia Systems Conference (ASMS) and 15th Signal Processing for Space Communications Workshop (SPSC), Berlin, Germany, 2018 .

[4] N. Alagha. 'Adjacent Beams Resource Sharing to Serve Hot Spots'. In 35th AIAA International Communications Satellite Systems Conference, International Communications Satellite Systems Conferences (ICSSC), Oct 2017.

[5] G. Taricco and A. Ginesi. Precoding for flexible high throughput satellites: Hot-spot scenario. IEEE Transactions on Broadcasting, pages 1-8, 2018.

terminals [6]. Alternatively, the performance of NCRS can be reduced [3].
[6] N. Noels, M. Moeneclaey, T. Ramírez, C. Mosquera, M. Caus, and Adriano Pastore. 'Symbol-asynchronous transmission in multibeam satellite user down-link: rate regions for novel superposition coding schemes'. In 2018 6th IEEE Global Conference on Signal and Information Processing, Anaheim, USA, 2018.

[7] A. Gholami Davoodi and S. A. Jafar. 'Transmitter Cooperation Under Finite Precision CSIT: A GDoF Perspective'. IEEE Transactions on Information Theory, 63(9):6020-6030, Sept 2017.

[8] J. Nocedal and S. J. Wright. Numerical Optimization. Springer, New York, NY, USA, second edition, 2006.

[9] Raj Jain, Dah-Ming Chiu, and W. Hawe. 'A quantitative measure of fairness and discrimination for resource allocation in shared computer systems'. tech. rep., Digital Equipment Corporation, DEC-TR-301, 1984. 